\title{
Delayed diagnosis and treatment of children with cancer during the COVID-19 pandemic
}

\author{
Michal Dvori ${ }^{1,2} \cdot$ Sarah Elitzur $^{1,2} \cdot$ Assaf Barg ${ }^{2,3} \cdot$ Shlomit Barzilai-Birenboim $^{1,2} \cdot$ Gil Gilad $^{1,2} \cdot$ Shirah Amar $^{1,2}$. \\ Helen Toledano ${ }^{1,2} \cdot$ Amos Toren $^{2,3} \cdot$ Sigal Weinreb $^{4} \cdot$ Gal Goldstein $^{4} \cdot$ Adi Shapira $^{5} \cdot$ Shifra Ash $^{5} \cdot$ Shai Izraeli $^{1,2}$. \\ Oded Gilad ${ }^{1,2}$
}

Received: 9 January 2021 / Accepted: 28 April 2021 / Published online: 18 June 2021

(C) Japan Society of Clinical Oncology 2021

\begin{abstract}
Background COVID-19, the novel coronavirus has caused a global pandemic affecting millions of people around the world. Although children, including children with cancer, have been found to be affected less commonly and less severely than adults, indirect effects of the pandemic on the diagnosis and treatment of children with cancer have been less described. Methods A survey was performed in the four largest tertiary pediatric hematology-oncology medical centers in Israel. Clinical and laboratory data were collected from the medical files of patients diagnosed or treated with cancer during April-October 2020.

Results Seventeen patients are described, who had a significant delay in diagnosis or treatment of cancer. These represent approximately $10 \%$ of all pediatric cancer diagnosed during the study period in these centers. A main cause of delay was fear of exposure to COVID-19 (fears felt by the patient, parent, physician, or decision-makers at the institution; or the implementation of national guidelines). Delays also resulted from co-infection with COVID-19 and the attribution of the oncologic symptoms to the infection. In addition, treatment was delayed of patients already diagnosed with cancer, due to COVID-19 infection detected in the patient, a family member, or a bone marrow donor.

Conclusion Fear from the COVID-19 pandemic may result in delayed diagnosis and treatment of children with cancer, which may carry a risk to dismal prognosis. It is crucial that pediatricians and patients alike remember that other diseases still prevail and must be thought of and treated in a timely fashion.
\end{abstract}

Keywords COVID-19 · Pediatric cancer · Delayed diagnosis · Delayed treatment

\section{Introduction}

Oded Gilad

dkgsguss@gmail.com

1 Department of Hematology-Oncology, Schneider Children's Medical Center of Israel, 14 Kaplan street, Petach Tikva, Israel

2 Sackler School of Medicine, Tel Aviv University, Tel Aviv, Israel

3 Department of Pediatric Hematology, Oncology and BMT, The Edmond and Lily Safra Children's Hospital, Sheba Medical Center, Ramat Gan, Israel

4 Department of Hematology-Oncology, Hadassah Hebrew University Medical Center, Jerusalem, Israel

5 Department of Pediatric Hematology-Oncology, Rambam Medical Center, and The Bruce Rappaport Faculty of Medicine, Technion-Israel Institute of Technology, Haifa, Israel
The novel coronavirus designated severe acute respiratory syndrome coronavirus 2 (SARs-CoV-2) was identified at the end of 2019 in China and rapidly spread, causing a global pandemic still active today, nearly 1 year later, termed Coronavirus Disease 2019 (COVID-19). As of October 2020, the World Health Organization (WHO) reports 43 million confirmed cases worldwide and over 1.15 million deaths due to the disease [1].

While the trends of the disease are still mostly obscure, children have been found to be affected less commonly than adults, and account for $1-8 \%$ of confirmed cases [2, 3]. Furthermore, infected children have generally shown a milder clinical course. For example, the hospitalization rate was 8 per 100,000 children in a large U.S. study [4]. More specifically, COVID-19 does not seem to have an effect 
on pediatric cancer patients, and those affected are mostly asymptomatic or with only mild disease [5].

As stated by Wolfs et al. [6], an important potential risk for cancer patients is delayed diagnosis and treatment during this pandemic. Delays in diagnosis could be due to decreased access of patients to their primary physicians, overuse of telemedicine-based-practice, avoidance of families to seek medical care due to fear of infection, cancellation of presumed non-essential laboratory and imaging tests, and cognitive biases resulting in physician misdiagnosis due to the pandemic. Therapy may be delayed due to preventive measures taken to protect the medical staff and other patients, and due to stem cell transplantation donors testing positive for COVID-19. The impact of COVID-19 on the management of adults with cancer has been described [7]. However, the impact on children with cancer is less known.

Here we present a series of pediatric cancer patients treated in major pediatric oncology centers in Israel, whose diagnosis or treatment was delayed due to the COVID-19 pandemic. This report should alert both specialists and general pediatricians to the need for prudence in diagnosing and treating children with cancer in a timely fashion, despite the ongoing COVID-19 pandemic.

\section{Methods}

A survey was performed in the four largest tertiary pediatric hematology-oncology medical centers in Israel, treating about 500 new cancer patients annually. We searched for pediatric patients treated during the months April-October 2020 whose diagnosis or treatment of their oncologic disease was delayed due to the COVID-19 pandemic. Clinical and laboratory data were collected from the patients' medical files. The study protocol was approved by the institutional review boards of Schneider Children's Medical Center of Israel and Rabin Medical Center.

\section{Results}

Seventeen pediatric cancer patients were identified, whose diagnosis or treatment of their oncologic disease was delayed due to the COVID-19 pandemic. Of them, nine were females; their ages ranged from 2 to 18 years (Table 1). Two were of Muslim Arab descent, one of Christian Arab descent, and the remaining were Jewish. This represents the ethnic composition of Israel. They were diagnosed with various oncologic diagnoses including acute lymphoblastic leukemia, acute myeloid leukemia, Hodgkin lymphoma, Burkitt lymphoma, diffuse large B cell lymphoma, Langerhans cell histiocytosis, Wilms tumor, osteosarcoma, rhabdomyosarcoma, Ewing sarcoma, nasopharyngeal carcinoma, and craniopharyngioma.

The patients could be classified into three subgroups according to the nature and cause of the delay (Table 1):

- Delay in diagnosis due to fear of exposure to COVID-19 (fear felt by the patient, parent, physician, or decisionmakers at the institution; or implementation of national guidelines).

- Delay in diagnosis of the oncologic disease due to coinfection with COVID-19 and the attribution of the oncologic symptoms to this infection.

- Delay in treatment of patients already diagnosed with an oncologic disease due to COVID-19 infection of the patient, a family member, or a bone marrow donor.

We hereby describe in depth four representative patients.

\section{Delayed cancer diagnosis due to the COVID-19 pandemic}

Patient 1 (Table 1) is a 14-year-old female of Jewish origin who complained of gradually worsening weakness and shortness of breath in effort (in time-even in minimal effort such as stepping on a chair). Her parents noticed a lack of appetite and weight loss of $5 \mathrm{~kg}$ in 3 months (10\% of her body weight). During that period, her parents contacted her primary care physician several times. They were told not to bring her to the clinic for a physical examination due to COVID-19 guidelines. At first, she was recommended psychological evaluation due to a presumed eating disorder. She was three times obligated to 2-week quarantines due to contact with persons who were infected with COVID19 and was denied arriving to the clinic for this reason. At one point, the patient developed fever and a cough and was instructed not to come to the clinic due to fear of spreading a potential COVID-19 infection. She was tested for COVID-19 several times and found negative.

Three months following her initial complaints, when supraclavicular lymphadenopathy developed, she was examined by her physician outside the clinic and was referred immediately to the emergency room. Upon arrival, her physical examination was positive for hepatosplenomegaly and diffuse lymphadenopathy. Chest X-ray, and later computed tomography, revealed a large mediastinal mass constricting a blood vessel and the trachea (superior mediastinal syndrome), a large left pleural effusion with a mass effect requiring immediate hospitalization in the intensive care unit, and chest drainage.

Biopsy from a supraclavicular enlarged lymph node matched the diagnosis of Hodgkin lymphoma. She is currently completing her induction treatment according to the 
Table 1 Characteristics of patients and the effect of COVID-19 on their diagnosis, treatment, and prognosis

\begin{tabular}{|c|c|c|c|c|c|}
\hline Case number & Age (years) & Sex & Ethnicity & Diagnosis & Brief description \\
\hline \multicolumn{6}{|c|}{ I. Delayed diagnosis due to fear of exposure to COVID-19 } \\
\hline 1 & 14 & Female & Jewish & Hodgkin lymphoma & $\begin{array}{l}3 \text { months delay in diagnosis due to the physi- } \\
\text { cian's refusal to examine }\end{array}$ \\
\hline 2 & 17 & Female & Jewish & Hodgkin lymphoma & $\begin{array}{l}\text { Several months delay in diagnosis due to the } \\
\text { physician's focus on a possible COVID-19 } \\
\text { diagnosis }\end{array}$ \\
\hline 3 & 5 & Male & Jewish & Craniopharyngioma & $\begin{array}{l}8 \text { months delay in diagnostic MRI due to can- } \\
\text { cellation of non-urgent examinations }\end{array}$ \\
\hline 4 & 3 & Female & Jewish & Metastatic Wilms tumor & $\begin{array}{l}1 \text { month delay in diagnosis due to the physi- } \\
\text { cian's refusal of imaging, for fear of exposing } \\
\text { the patient to COVID-19 }\end{array}$ \\
\hline 5 & 15 & Female & Muslim Arab & Relapse metastatic osteosarcoma & $\begin{array}{l}6 \text { months delay in diagnosis of relapse due to } \\
\text { the parent's refusal of imaging }\end{array}$ \\
\hline 6 & 15 & Male & Jewish & Acute myeloid leukemia & $\begin{array}{l}2 \text { months delay in diagnosis due to fear of seek- } \\
\text { ing medical care }\end{array}$ \\
\hline 7 & 14 & Male & Jewish & Rhabdomyosarcoma & $\begin{array}{l}3 \text { months delay in diagnosis due to physician' } \\
\text { refusal of examination, for fear of exposing } \\
\text { the patient to COVID-19 }\end{array}$ \\
\hline 8 & 2 & Female & Jewish & Acute myeloid leukemia & $\begin{array}{l}1 \text { month delay in diagnosis due to medical care } \\
\text { based on telemedicine and a delay in referral } \\
\text { for blood tests }\end{array}$ \\
\hline 9 & 18 & Male & Jewish & Ewing sarcoma & $\begin{array}{l}6 \text { months delay in diagnosis due to symptoms } \\
\text { (fever and cough) appearing while abroad, } \\
\text { fear of flying back, and difficulty finding a } \\
\text { flight }\end{array}$ \\
\hline 10 & 14 & Male & Jewish & Nasopharyngeal carcinoma & $\begin{array}{l}6 \text { months delay in diagnosis due to the } \\
\text { physician's focus on a possible COVID-19 } \\
\text { diagnosis (loss of smell sense as a presenting } \\
\text { symptom) }\end{array}$ \\
\hline 11 & 11 & Male & Jewish & Diffuse large B cell lymphoma & $\begin{array}{l}2 \text { months delay in diagnosis due to the family's } \\
\text { fear of coming to the hospital to perform } \\
\text { recommended biopsy of lymph nodes }\end{array}$ \\
\hline 12 & 3 & Female & Jewish & Acute lymphoblastic leukemia & $\begin{array}{l}1.5 \text { months delay in diagnosis due to the fam- } \\
\text { ily's fear of seeking medical care }\end{array}$ \\
\hline \multicolumn{6}{|c|}{ II. Misdiagnosis of oncologic disease as COVID-19 } \\
\hline 13 & 9 & Male & Jewish & Burkitt lymphoma & $\begin{array}{l}\text { 1-month delay in diagnosis due to symptoms } \\
\text { attributed to a present COVID-19 infection }\end{array}$ \\
\hline 14 & 18 & Male & Jewish & Hodgkin lymphoma & $\begin{array}{l}5 \text { weeks delay in diagnosis due to symptoms } \\
\text { attributed to a present COVID-19 infection }\end{array}$ \\
\hline \multicolumn{6}{|c|}{ III. Treatment Impaired due to COVID-19 infection or exposure } \\
\hline 15 & 15 & Female & Jewish & $\begin{array}{l}\text { Acute myeloid leukemia on the back- } \\
\text { ground of myelodysplastic syndrome }\end{array}$ & $\begin{array}{l}\text { More than } 2 \text { months delay in hematopoietic } \\
\text { stem cell transplantation due to COVID- } \\
19 \text { exposure of a donor. Leukemic blasts } \\
\text { returned prior to transplantation, worsening } \\
\text { the prognosis }\end{array}$ \\
\hline 16 & 13 & Female & Muslim Arab & Hodgkin lymphoma & $\begin{array}{l}2 \text { weeks delay in treatment due to COVID-19 } \\
\text { positive status of the patient prior to central } \\
\text { line insertion and fertility preservation } \\
\text { surgery }\end{array}$ \\
\hline 17 & 18 & Female & Christian Arab & Langerhans cell histiocytosis reactivation & $\begin{array}{l}\text { 1-month delay in chemotherapy treatment due } \\
\text { to COVID-19 positive status of the patient } \\
\text { and her mother }\end{array}$ \\
\hline
\end{tabular}


EuroNet-PHL-C2 protocol and is still hospitalized with chylothorax secondary to the mediastinal mass.

\section{Delayed diagnosis of relapsed disease due to the COVID-19 pandemic}

Patient 5 (Table 1), a 15-year-old female of Muslim Arab origin, was successfully treated for right distal femur osteogenic sarcoma. After remission, she was followed at the recovering patients' clinic. One year into remission, routine follow-up computed tomography of the lungs without contrast material revealed a finding in the spleen that raised suspicion for metastasis or a hemangioma. She was requested to perform a triphasic abdominal CT to better characterize the finding. Afraid of being infected with COVID-19, the patient did not show up to the scheduled CT scan and failed to make another appointment.

Six months later, the patient presented with nonspecific pain. Abdominal CT results were compatible with a spleen hemangioma, but the chest CT demonstrated two lung metastases that underwent surgical resection, together with a right middle lobectomy. She is now post-operation.

Diagnoses of 12 of the 17 patients (1-12 in Table 1) were delayed due to fear of exposure to COVID-19. Most of the delays were due to physicians' refusal of face-to-face examination, refusal or delay in imaging, or cognitive biases ("anchoring bias") leading the physicians to focus on possible COVID-19 diagnosis during the pandemic and not widen the differential diagnosis. Some of the delayed diagnoses were due to parents' fear of seeking medical care during the pandemic. Ten patients are currently under treatment, one patient (patient 4, has already finished treatment and is now in remission, and one patient (patient 9) died during induction.

\section{Oncologic diagnosis masked by concurrent COVID-19 infection}

Patient 13 (Table 1), a 9-year-old male of Jewish origin, complained of abdominal pain and a swollen abdomen. Following his father's diagnosis with COVID-19, the patient was tested and found positive. He later had a fever, diarrhea, increased abdominal swelling, and jaundice. During that period, the patient was at home in quarantine, according to the Israeli pandemic guidelines. His parents communicated with physicians dedicated to treat home quarantine COVID19 patients during the pandemic through telemedicine. They expressed their concern regarding his symptoms but reassured that his symptoms were apparently all manifestations of COVID-19 infection. After 1 month of worsening symptoms, his mother noticed skin and conjunctival jaundice and sent a picture to his pediatrician who referred him urgently to the emergency department.
Upon arrival, the patient was diagnosed with a large right pleural effusion requiring drainage, hepatitis, cholestasis, jaundice with direct hyperbilirubinemia up to $9.8 \mathrm{mg} /$ $\mathrm{dL}$, pancreatitis, increased abdominal pressure, and early signs compatible with a tumor lysis syndrome, including acute renal failure. Serology for COVID-19 was at that point already negative. Computed tomography demonstrated bilateral retrosternal masses, upper abdominal masses, enlarged lymph nodes in the mesentery and liver porta, ascites, and extra-hepatic and intra-hepatic bile-duct widening due to mass effect and pressure on the common bile duct. Cytology from the pleural fluid revealed the diagnosis of Burkitt lymphoma. The patient was admitted to the pediatric intensive care unit and steroid treatment was begun accordingly. He is currently after his first chemotherapy course.

The diagnoses of oncologic disease of two patients (13, 14 in Table 1) were delayed due to simultaneous infection with COVID-19 and attribution of the oncologic symptoms to this infection. For both patients, a significant delay in the diagnosis occurred; they are both currently under treatment.

\section{Delay in treatment due to the COVID-19 pandemic}

Patient 15, a 15-year-old female of Jewish origin, was diagnosed with acute myeloid leukemia following 1 month of weakness and 2 weeks of light-headedness. She presented with pancytopenia with hemoglobin of $2.9 \mathrm{~g} / \mathrm{dL}$, platelets $3000 * 10^{9} / \mathrm{L}$, and $1.7 * 10^{9} / \mathrm{L}$ leucocytes with $0.07 * 10^{9} / \mathrm{L}$ neutrophils. Bone marrow cytogenetics revealed $7 \mathrm{q}$ deletion. Due to the karyotype, extremely low counts upon diagnosis and the large MCV (107fL), myelodysplastic syndrome as a basis for acute myeloid leukemia was suspected. Indeed, in a custom-made targeted new generation sequencing panel, a GATA2 germline mutation was diagnosed (the patient did not share any of the symptoms commonly seen in Emberger syndrome). The decision was reached to treat her with hematopoietic stem cell transplantation (HSCT) as soon as possible after the suppression of her leukemia by induction chemotherapy. Three of her siblings were found as HLA genotypically matched potential donors. Prior to the admission for HSCT conditioning, both her parents tested positive for COVID-19. The patient herself was negative for COVID19 and was transferred to a COVID-19 unit for quarantine. One week later, two of her three potential matched siblings were found positive for COVID-19. Though the third sibling tested negative, he had to enter quarantine for 2 weeks due to his exposure to his affected family members. Consequently, the patient's HSCT was postponed by almost 1 month. While waiting for the clearance of her donor, she developed a severe infection for which she is currently being treated. Her HSCT has now been delayed by 2 months and she faces a significant risk for recurrence of the leukemic phase and potential treatment failure. 
Treatment was delayed due to a COVID-19 infection for three patients (patients 15-17 in Table 1) who were diagnosed with an oncologic disease. For one, HSCT was delayed due to COVID-19 exposure of the donor. For another, treatment was delayed due to the patient being identified as COVID-19 positive in routine screening prior to a central line insertion and fertility preservation surgery. Her surgery was postponed by 2 weeks since she was not in a critical state, and her treatment was postponed accordingly. Treatment was delayed for the third patient as she and her mother were identified as being COVID-19 positive. Patient 15 is now being prepared for her HSCT, patients 16 and 17 are currently under treatment.

\section{Discussion}

Until now, COVID-19 has been found to affect children less commonly than adults, and to cause a milder course in children [2-4]. Furthermore, published reports confirm little direct effect of COVID-19 on pediatric oncological patients $[5,6]$.

Despite the above, to the best of our knowledge, there are no case reviews regarding the indirect effects of the COVID19 pandemic on the diagnosis and treatment of children with cancer. Two "letters to the editor" addressed this topic. Offenbacher et al. [11] reported a decrease in diagnoses of pediatric solid tumors during the COVID-19 pandemic, a delay that correlated with advanced disease at diagnosis. Parasole et al. [12] described three children (two of them died) who arrived at the hospital in critical condition at the onset of acute lymphoblastic leukemia, most likely as a consequence of fear of COVID-19. Those reports, together with the current series, demonstrate that delays in cancer diagnoses during the COVID-19 period may be more hazardous than the direct effects of the pandemic. Specifically, social distancing, quarantine guidelines, and the reliance on telemedicine, in contrast to face-to-face medical encounters, has decreased the availability of non-urgent medical services. Moreover, cognitive biases that direct physicians' diagnosis to COVID-19, as the most spoken-about medical condition, may lead to dangerous delays in diagnosis and in treatment of pediatric oncological patients.

Here we reported seventeen pediatric oncological patients with delays in diagnosis or treatment due to the COVID19 pandemic. The phenomenon crossed ages, ethnic background, and diagnoses. Delays were as long as 8 months. During the period of our survey about 200 new children with cancer were treated in the participating centers. Thus, diagnosis and treatment has been significantly delayed in up to $10 \%$ of all children with cancer.

Questions arise as to whether delays in cancer diagnoses are meaningful, and as to the effects on prognosis.
In osteosarcoma, for example, delays in diagnosis have been shown not to correlate with the likelihood of primary metastases, initial invasion, and possibly survival $[8,9]$. In their review, Brasme et al. [10] reached similar conclusions regarding central nervous system (CNS) tumors and Ewing sarcoma. But the timely diagnosis of lung metastases, such as in the patient described here, is critical. Surgery is the only approach that extends life in patients with osteosarcoma. Delayed diagnosis may render the metastatic lesion unresectable and, therefore, have a significant impact on survival. However, the relation between time to diagnosis and poor outcome for retinoblastoma is well established. Time to diagnosis apparently associates inversely with survival for leukemia, nephroblastoma, and rhabdomyosarcoma, although due to the sparse data, this relation is uncertain. Similarly, data are insufficient for Hodgkin and non-Hodgkin lymphoma, neuroblastoma, and germ-cell tumors.

The abovementioned counterintuitive findings, especially in CNS tumors, osteosarcoma, and Ewing sarcoma, are explained by the rationale that tumors that are more aggressive are diagnosed earlier than those that are more indolent, and because they are more aggressive their prognosis is accordingly poorer. This, therefore, explains delays in diagnosis in "normal" eras. However, little is known as to whether delays in diagnosis that are not due to the nature of the oncologic disease, such as delays during the COVID19 pandemic period, will have similar effects. Moreover, while effective chemotherapy may overcome the potential deleterious effects of delayed diagnosis, delays such as those described here, are associated with a substantial increase in life-endangering complications due to the diagnosis of cancer in advanced stages. Examples of such are respiratory and cardiovascular compromise by chest tumors, spontaneous tumor lysis syndrome, and more.

Interestingly, the distribution of the underlying malignancies in this patient series is not representative of the frequency of occurrence of pediatric cancers in the general population (the distribution of pediatric cancer types in Israel is similar to that of the economically developed world). Cancer type may be an important factor related to diagnosis delay. Timely diagnosis may be affected by tumor growth-rate and aggressiveness, its anatomic site, and clinical presentation. A shorter delay would be expected in tumors presenting with rare symptoms versus those presenting with common, nonspecific symptoms. It is also possible that older patients, especially adolescents, are at higher risk of delayed diagnosis. Thus, for example, acute lymphoblastic leukemia (ALL), the most common childhood malignancy (accounts for approximately one-quarter of all childhood malignancies in Israel, according to the Israeli cancer society, http://www.cancer.org.il), is underrepresented in our study because its diagnosis is less likely to be delayed, due 
to its peak incidence at lower ages (2-4 years) and rapid growth-rate.

\section{Conclusion}

It seems plausible that the COVID-19 pandemic influences pediatric cancer patients in several indirect ways, which may carry a risk to disease prognosis. For as long as this pandemic is with us, it is crucial that pediatricians and patients alike remember that other diseases still prevail and must be thought of and treated in due time.

Acknowledgements We thank Ms. Cindy Cohen for her editing of the manuscript.

\section{Declarations}

Conflict of interest All authors have no conflict of interest.

\section{References}

1. World Health Organization (WHO) Coronavirus Disease (COVID19) dashboard. https://covid19.who.int. Accessed 26 Oct 2020

2. Posfay-Barbe KM, Wagner $\mathrm{N}$ et al (2020) COVID-19 in children and the dynamics of infection in families. Pediatrics 146(2):e20201576. https://doi.org/10.1542/peds.2020-1576

3. Docherty AB, Harrison EM et al (2020) Features of 20133 UK patients in hospital with COVID-19 using the ISARIC WHO
Clinical Characterisation Protocol: prospective observational cohort study. BMJ 369:m1985

4. Kim L, Hitaker M et al (2020) Hospitalization rates and characteristics of children aged $<18$ years hospitalized with laboratoryconfirmed COVID-19 - COVID-NET, 14 States, March 1-July 25, 2020. MMWR Morb Mortal Wkly Rep 69(32):1081

5. Hrusak O, Kalina T et al (2020) Flash survey on severe acute respiratory syndrome coronavirus- 2 infections in paediatric patients on anticancer treatment. Eur J Cancer 132:11-16

6. Wolfs TFW, Attarbaschi A et al (2020) COVID-19 - impact on childhood haematology patients. Hemasphere 4(5):e465

7. Hamilton W (2020) Cancer diagnostic delay in the COVID-19 era: what happens next? Lancet Oncol 8:1000-1002

8. Martin S, Ulrich C et al (2007) Delays in cancer diagnosis in underinsured young adults and older adolescents. Oncologist 12:816-824

9. Goyal S, Roscoe J et al (2004) Symptom interval in young people with bone cancer. Eur J Cancer 40:2280-2286

10. Brasme JF, Morfouace M et al (2012) Delays in diagnosis of paediatric cancers: a systematic review and comparison with expert testimony in lawsuits. Lancet Oncol 13(10):e445-459

11. Offenbacher R, Knoll M, Loeb DM (2020) Delayed presentations of pediatric solid tumors at a tertiary care hospital in the Bronx due to COVID-19. Pediatr Blood Cancer 68(2):e28615. https:// doi.org/10.1002/pbc.28615

12. Parasole R, Stellato P, Conter V et al (2020) Collateral effects of COVID-19 pandemic in pediatric hematooncology: fatalities caused by diagnostic delay. Pediatr Blood Cancer 67(8):e28482

Publisher's Note Springer Nature remains neutral with regard to jurisdictional claims in published maps and institutional affiliations. 